\title{
Open-source Simulation of Underwater Gliders
}

\author{
Davide Grande ${ }^{(\mathbb{1}}$ \\ Department of Mechanical Engineering \\ University College London \\ London, UK \\ davide.grande.19@ucl.ac.uk
}

Peng $\mathrm{Wu}^{(10}$

Department of Mechanical Engineering

University College London

London, UK

\author{
Luofeng Huang ${ }^{(}$ \\ Department of Mechanical Engineering \\ University College London \\ London, UK
}

\author{
Giles Thomas ${ }^{\circledR}$ \\ Department of Mechanical Engineering \\ University College London \\ London, UK
}

\author{
Catherine A. Harris ${ }^{(0)}$ \\ Marine Autonomous and Robotic Systems \\ National Oceanography Centre \\ Southampton, UK
}

\author{
Enrico Anderlini ${ }^{\circledR}$ \\ Department of Mechanical Engineering \\ University College London \\ London, UK
}

\begin{abstract}
Autonomous underwater gliders (AUGs) are currently deployed in oceans throughout the globe and are recording real-time, in-situ data. Simulating AUGs is rendered particularly difficult by the identification of the underlying dynamic model, as these vehicles embed several internal movable components. Acausal simulators can significantly improve the possibility to study and understand the dynamics of this class of vehicles and can in turn support the design of more robust control systems. In this paper, an open-source simulator architecture designed in OpenModelica is proposed to simulate underwater gliders. The validation is carried out using two different AUGs models, a ROGUE and a Seawing. The vehicle dynamics is firstly compared with analytical results and, following, with values obtained by means of another simulator. Further steps will entail comparing the dynamics of a simulated Seaglider with real deployment data publicly available. In this work, some of the main hydrodynamic and geometrical properties of a Seaglider are identified, computed through Computational Fluid Dynamics (CFD) analyses and retrieved from the mission ballast sheet. Overall, the developed model is expected to enhance gliders' control strategies, thus improving their performance and mitigating incidents such as being carried away by undesired ocean currents.
\end{abstract}

Index Terms-Modelica, Autonomous Underwater Gliders, Seaglider, Multibody Dynamics

\section{INTRODUCTION}

Autonomous underwater gliders (AUGs) are increasingly being used by research centres to map, monitor and study the oceans. Unlike standard Autonomous Underwater Vehicles (AUV), which use thrusters and control surfaces to navigate, the gliders use a combination of internal moving masses and a Variable Buoyancy Device (VBD) as actuators. The gliders typically operate by diving in a characteristic sawtooth pattern in the vertical plane. The dive entails increasing either the internal mass or reducing the vehicle volume using the VBD, and consequently shifting forward the centre of mass. Once the descending part of the path is completed, the internal moving mass is shifted towards the rear of the vehicle and the overall mass of the vehicle is reduced or the volume increased, inducing the glider to ascend back to the surface.

D. Grande's studentship was supported by the National Oceanography Centre and by the University College London.
The gliders present substantial advantages when compared to thruster-propelled AUVs in terms of energy consumption and mission endurance [1]. On the other hand, the internal movable masses, the VBD and the depths reached during each dive introduce modelling complexity. Additionally, during each mission, which can last up to several months, the gliders cover long distances and are exposed to a large variety of unplanned and undesired conditions that can jeopardise the mission success. Several commercial gliders have been developed in the last decades, which include the Seaglider [2], Slocum [3] and Seawing [4]. Despite showing different configurations and positions of the control actuators, the gliders share common causes of faults linked to mechanical, logistical and environmental causes [5].

Developing accurate dynamic models and in turn simulators, can support the analysis, forecast and mitigation of the faults by testing more robust control strategies. Dynamic models of gliders have been developed in [6]-[8]. On top of the highly nonlinear effects characterising the interaction between the AUGs and the marine environments, the dynamics of the vehicles is rendered particularly complex by the presence of internal movable components. AUGs are in fact multibody mechanical systems composed of the hull, the internal shifting mass, the variable ballast mass and in some models of an additional internal rolling mass. These actuators significantly increase the complexity of modelling the systems compared to the standard 6 Degrees Of Freedom (DOF) models typically used for manoeuvring studies. Depending on the presence of the internal rolling mass, these vehicles can be modelled as 8 or 9 DOF systems.

The aim of this paper is to design and validate an opensource simulator for underwater gliders to be later used to improve the onboard control system. Additionally, the simulation can be used to analyse the effect of undesirable environmental factors, such as ocean currents or collision with drifting debris. OpenModelica is deemed as the best option being specifically designed for multibody mechanical systems. As compared with the traditional casual simulation approach, OpenModelica offers acausal resolution algorithms capable of 
substantially speeding up the simulation times of complex dynamical systems.

The simulator is designed starting from the multibody mechanical library of OpenModelica and adapting some components to the underwater application, extending the work previously proposed in [9]. The architecture is composed of several masses representing the hull, the movable masses, the VBD, and the steering surfaces, and by applying the hydrostatic and hydrodynamic forces at the interfaces.

The validation of the simulator is carried out in two steps of increasing complexity, entailing the comparison of the obtained results firstly with an analytical model and following with the output of another simulator. Future steps will include the comparison against the real deployment data of a Seaglider owned by the National Oceanography Centre of Southampthon, UK.

Differently from the Slocum and Seawing, the full set of hydrodynamic and geometrical parameters is not available in the literature. The main hydrodynamic and geometric parameters are reported in this work. Computational Fluid Dynamics (CFD) analyses are carried out to identify the main hydrodynamic coefficients, while the mechanical properties of the vehicle are obtained from trim and ballast sheets, and by means of CAD drawings.

The paper is organised as follows: Section II derives the dynamic model of the AUGs, Section III reports the architecture of the simulator, Section IV illustrates the two-steps procedures used to validate the model and collects the results of the CFD and the ballast sheet analyses. Conclusion and future work are reported in Section V.

\section{GLIDER MODEL}

Three reference frames are typically used when modelling AUGs: the inertial, the body and the flow frame [6], [8]. The inertial frame is a non-accelerating frame that can be set to be Earth-fixed [10]. For this work, a North-East-Down (NED) frame of origin $\left\{O_{i}\right\}$ is selected as inertial since the distances involved in the simulations proposed are of small scale as compared to the Earth radius. Care must be taken when long missions are simulated, as other frames that account for the Earth rotation, such as the Earth-centred Earth-fixed (ECEF), might be more appropriate. The body frame origin $\left\{O_{b}\right\}$ is fixed at the centre of buoyancy of the hull, which is assumed to be geometrically fixed during the dives. The $x_{b}$-axis of the body frame coincides with the longitudinal axis of the vehicle and the $z_{b}$-axis points downward. Following, as the hydrodynamic forces and moments depend on the relative velocity and orientation of the vehicle with respect to the surrounding fluid, a third frame, defined as flow frame, is introduced. The origin $\left\{O_{f}\right\}$ of the flow frame is coincident with the origin of the body frame, and its orientation is obtained starting from the latter one through two sequential rotations of the angles $\alpha$ and $\beta$, with the convention reported in Figure 1.

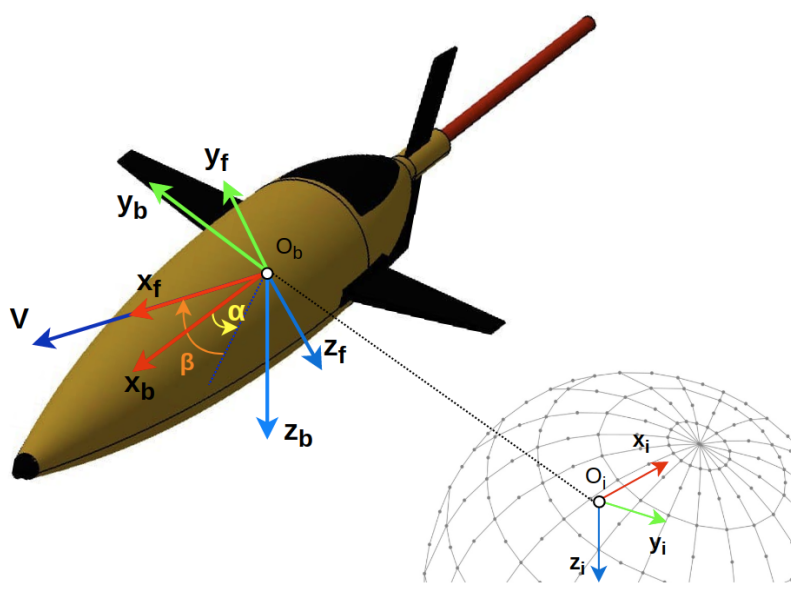

Fig. 1: Reference frames used for modelling of an AUG (Seaglider - ogive model).

Under the assumption of steady ocean currents, the magnitude of the flow speed vector is defined as [10]:

$$
V=\sqrt{u^{2}+v^{2}+w^{2}}
$$

where $u, v$ and $w$ represent the three components of the vehicle body speed with respect to the inertial frame, in accordance with the Society of Naval Architects and Marine Engineers (SNAME) nomenclature.

Additionally, the hydrodynamic angles $\alpha$ and $\beta$, defined as angle of attack and sideslip angle, respectively, are computed as:

$$
\begin{aligned}
& \alpha=\tan ^{-1}\left(\frac{w}{u}\right) \\
& \beta=\sin ^{-1}\left(\frac{v}{V}\right)
\end{aligned}
$$

The hydrodynamic forces act on the centre of pressure of the vehicle, that do not coincide with the centre of the body frame $\left\{O_{b}\right\}$. The separation between the two centres gives rise to a moment around the origin of the body frame.

The hydrodynamic force and moment vectors in the flow frame can thus be expressed as reported in [8]:

$$
\begin{aligned}
& \boldsymbol{F}_{\boldsymbol{h d}}^{\boldsymbol{f}}=\left[\begin{array}{lll}
-D & S F & -L
\end{array}\right]^{T} \\
& \boldsymbol{M}_{\boldsymbol{h \boldsymbol { d }}}^{\boldsymbol{f}}=\left[\begin{array}{lll}
T_{D L 1} & T_{D L 2} & T_{D L 3}
\end{array}\right]^{T}
\end{aligned}
$$

where he components of drag, lift, side-force and the corresponding moments, can be expressed in the quasi-steady state form as:

$$
\begin{aligned}
& D \approx\left(K_{D 0}+K_{D} \alpha^{2}\right) V^{2} \\
& S F \approx K_{\beta} \beta V^{2} \\
& L \approx\left(K_{L 0}+K_{L} \alpha\right) V^{2} \\
& T_{D L 1} \approx\left(K_{M R} \beta+K_{p} p\right) V^{2} \\
& T_{D L 2} \approx\left(K_{M 0}+K_{M} \alpha+K_{q} q\right) V^{2} \\
& T_{D L 3} \approx\left(K_{M Y} \beta+K_{r} r\right) V^{2}
\end{aligned}
$$


The hydrodynamic coefficients $\left(K_{i}\right)$ appearing in Equation (4) depend on the specific geometry of the vehicle.

In Section IV-C, the assumption that the quasi-steady state approximation of the hydrodynamic vectors is sufficient to characterise the motion of the gliders, is validated.

The $\boldsymbol{F}_{\boldsymbol{h} \boldsymbol{d}}^{\boldsymbol{f}}$ and $\boldsymbol{M}_{\boldsymbol{h} \boldsymbol{d}}^{\boldsymbol{f}}$ vectors can be rotated into the body frame by means of the following rotation matrix:

$$
\boldsymbol{R}_{\boldsymbol{f}}^{b}=\left[\begin{array}{ccc}
\cos \alpha \cos \beta & -\cos \alpha \sin \beta & -\sin \alpha \\
\sin \beta & \cos \beta & 0 \\
\sin \alpha \cos \beta & -\sin \alpha \sin \beta & \cos \alpha
\end{array}\right]
$$

Moreover, added mass effects arise due to the exchange of inertia between a vehicle in motion and the surrounding fluid [9]. As the added mass coefficients depend on the geometry of the vehicle, in Section IV-C a procedure is illustrated to compute these parameters based on the assumption that the glider's hull can be approximated as a prolate spheroid.

The hydrostatic forces, namely buoyancy and gravity, generate a moment with respect to the origin of the body frame depending on the choice of the location of the latter. For underwater vehicles applications, the origin of the body frame $\left\{O_{b}\right\}$ can be chosen coincident with the centre of buoyancy. When modelling AUGs, the buoyancy force can be split in two contributions, one due to the constant volume of the vehicle $\left(B_{h}\right)$, acting on the hull volume centroid, and a second one generated by the VBD, acting on the centroid of the VBD $\left(B_{V}\right)$. The notation reported in [9], can be extended as:

$$
\begin{aligned}
& W=m g \\
& B_{h}=\rho g \nabla_{h} \\
& B_{V}=\rho g \nabla_{V}
\end{aligned}
$$

where: $\rho$ represents the water density, $m$ the overall mass of the vehicle, $\nabla_{h}$ the volume of the hull and $\nabla_{V}$ the volume of the VBD. The hydrostatic forces in the inertial frame can be summarised as:

$$
\begin{aligned}
\boldsymbol{f}_{\boldsymbol{g}}^{\boldsymbol{i}} & =\left[\begin{array}{lll}
0 & 0 & W
\end{array}\right]^{T} \\
\boldsymbol{f}_{\boldsymbol{h}}^{\boldsymbol{i}} & =\left[\begin{array}{lll}
0 & 0 & -B_{h}
\end{array}\right]^{T} \\
\boldsymbol{f}_{\boldsymbol{V}}^{\boldsymbol{i}} & =\left[\begin{array}{lll}
0 & 0 & -B_{V}
\end{array}\right]^{T}
\end{aligned}
$$

The hydrostatic forces can be rotated from the inertial to the body frame by means of Euler angles or quaternion parametrisation.

Introducing $\boldsymbol{r}_{\boldsymbol{g}}^{\boldsymbol{b}}, \boldsymbol{r}_{\boldsymbol{h}}^{\boldsymbol{b}}$ and $\boldsymbol{r}_{\boldsymbol{V}}^{\boldsymbol{b}}$ as the vectors collecting the distances from $\left\{O_{b}\right\}$ to the centre of mass, centre of buoyancy of the hull and centre of buoyancy of the VBD, respectively, it is possible to summarise the hydrostatic force and torque vector in body frame as:

$$
g^{b}=-\left[\begin{array}{c}
f_{g}^{b}+f_{h}^{b}+f_{V}^{b} \\
r_{g}^{b} \times f_{g}^{b}+r_{h}^{b} \times f_{h}^{b}+r_{V}^{b} \times f_{V}^{b}
\end{array}\right]
$$

The complete expression of the hydrostatic vector rotated in the body frame is reported in [11].

\section{SimUlator ARCHITECTURE}

Modelica $^{1}$, born in 1996 [12], is an open-source objectoriented modelling language. Similarly to Simscape, it offers libraries to model mechanical, electrical, electronic, hydraulic, thermal, and control components, but with the advantage that, being open-source, the packages are fully customisable. Different Integrated Development Environments (IDEs) for Modelica are available, including Dymola and OpenModelica. Dymola , developed by Dassalut Systèmes, represents a widely used paid option, while OpenModelica is deemed as the most complete open-source development environment [13].

Moreover, Modelica offers standard communication interfaces to interact with other software or programming languages, such as Python, or middleware, such as Robot Operating System (ROS). This renders it an optimal open-source choice for mixed architectures, exploiting the best functionalities of each component, such as designing the system dynamics in Modelica, the control laws in Python or $\mathrm{C}++$, and the testing of real-time performances in ROS.

No library is specifically available for underwater vehicles but the Modelica language has already been used to simulate multibody underwater vehicles in [9] and space vehicles in [14].

The simulator architecture proposed in this work follows the structure illustrated in [9]: the vehicle is designed as a multibody system by linking components from the MechanicsMultiBody library through prismatic and 3D revolute joints. As reported in Figure 2, the system comprises 6 main rigid bodies representing the hull, the sliding, the rolling, the offset, the ballast and the VBD masses. Two bodies representing the rudder and stern planes are added for modularity.

Following, three sets of external forces and torques are integrated into the simulator and are applied to the glider subcomponents through the mechanical interfaces of the Multibody library. The hydrodynamic force and moment vectors are rotated in the body frame and applied to its origin $\left\{O_{b}\right\}$. The added mass forces and torques, are also applied to $\left\{O_{b}\right\}$ as purely dependent on the body linear and angular accelerations. Both glider speeds and accelerations are retrieved by means of virtual sensors. The buoyancy force and the corresponding torques are applied to the centre of buoyancy while the gravity force acts on the entire simulation space as a uniformly distributed force field.

For the initial simulator design, the water currents are assumed to be steady, consistently with the model described in Section II.

\section{SimUlator VALIDATION}

The simulator has been verified and validated in two steps of increasing complexity. The results generated by the proposed simulator are compared against published results for two different vehicle types whose model parameters are available in the literature. Further comparisons will be carried out with real deployment data.

\footnotetext{
${ }^{1}$ https://www.modelica.org/
} 


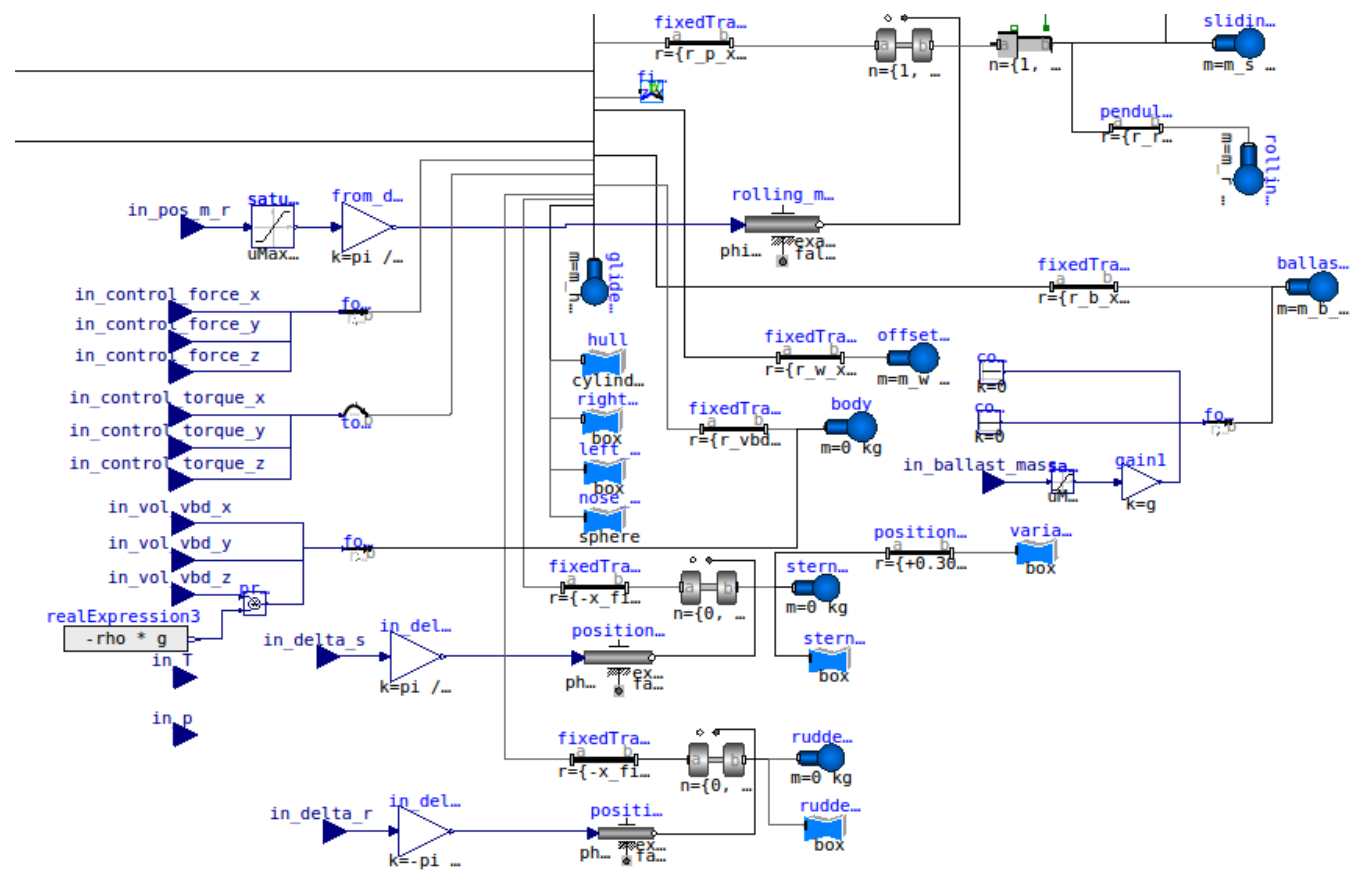

Fig. 2: OpenModelica - multibody architecture.

\section{A. ROGUE glider}

The ROGUE glider, described in [6], is the first chosen vehicle to investigate purely vertical dynamics. The expression of the quasi-steady hydrodynamic forces and moments in the vertical plane can be obtained by simplifying Equations (3) and (4) as:

$$
\begin{aligned}
& \boldsymbol{F}_{\boldsymbol{h d}}^{\boldsymbol{f}}=\left[\begin{array}{lll}
-D & 0 & -L
\end{array}\right]^{T} \\
& \boldsymbol{M}_{\boldsymbol{h d}}^{\boldsymbol{f}}=\left[\begin{array}{lll}
0 & T_{D L 2} & 0
\end{array}\right]^{T}
\end{aligned}
$$

where:

$$
\begin{array}{r}
D \approx\left(K_{D 0}+K_{D} \alpha^{2}\right)\left(u^{2}+w^{2}\right) \\
L \approx\left(K_{L 0}+K_{L} \alpha\right)\left(u^{2}+w^{2}\right) \\
T_{D L 2} \approx\left(K_{M 0}+K_{M} \alpha\right)\left(u^{2}+w^{2}\right)
\end{array}
$$

To verify the simulator, four different sets of inputs are commanded to test the gliding dynamics of the vehicles; following, the results are compared with the ones obtained in [6]. The two ascending and the two descending sections of the paths are reported in Figure 3, while the 3D rendering of the vehicle is illustrated in Figure 4.

In Table I, the results of the first and fourth sections of the glide are compared (the second and third ones are symmetrical). It is worth recalling that the results reported in [6] are analytically obtained from the dynamic model, proving that the proposed approach is capable of reproducing the same results with a maximum relative error bounded below $5 \%$ with respect to the theoretical expected values.

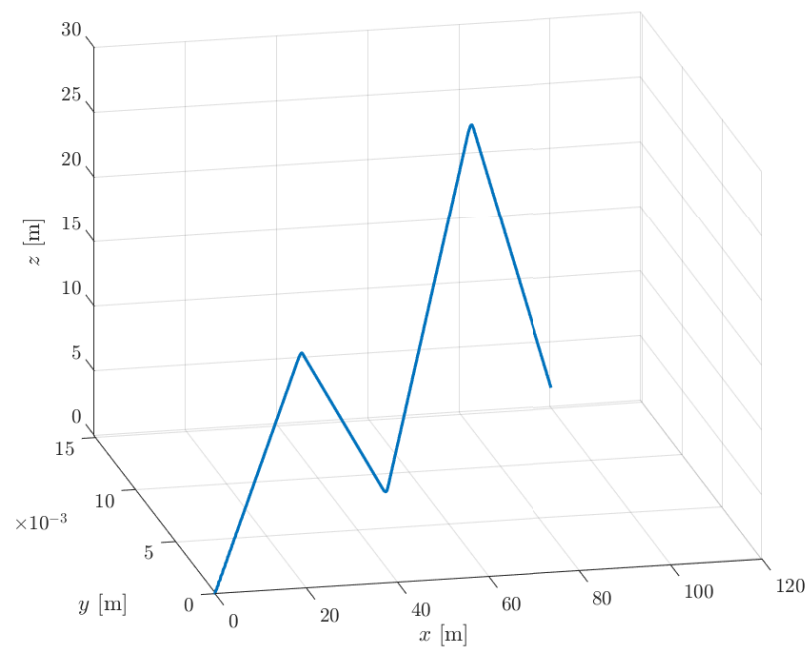

Fig. 3: ROGUE trajectory from the OpenModelica simulations. 


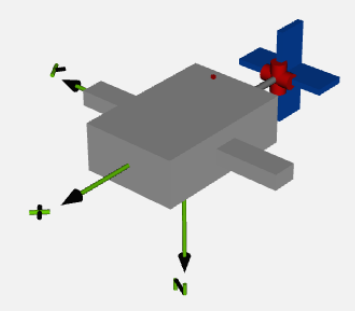

Fig. 4: OpenModelica rendering of the ROGUE glider.

TABLE I: Theoretical vs. simulation results.

\begin{tabular}{cccc}
\hline Quantity & $\begin{array}{c}\text { ROGUE } \\
\text { reference [6] }\end{array}$ & OpenModelica & Relative error \\
\hline Down $30^{\circ}$ & & & \\
\hline$\xi\left[^{\circ}\right]$ & -30.00 & -30.18 & $0.60 \%$ \\
$\theta\left[^{\circ}\right]$ & -23.00 & -23.97 & $4.22 \%$ \\
$\alpha\left[^{\circ}\right]$ & 6.30 & 6.21 & $1.43 \%$ \\
$V[\mathrm{~m} / \mathrm{s}]$ & 0.30 & 0.30 & $0.0 \%$ \\
\hline $\mathrm{Up} 45^{\circ}$ & & & \\
\hline$\xi\left[^{\circ}\right]$ & 45.00 & 45.12 & $0.27 \%$ \\
$\theta\left[^{\circ}\right]$ & 41.50 & 41.70 & $0.48 \%$ \\
$\alpha\left[^{\circ}\right]$ & -3.50 & -3.42 & $2.29 \%$ \\
$V[\mathrm{~m} / \mathrm{s}]$ & 0.37 & 0.37 & $0.0 \%$ \\
\hline
\end{tabular}

Where:

- $\theta$ is the pitch angle;

- $\alpha$ is the angle of attack;

- $\xi$ is defined as the glide path angle $(\xi=\theta-\alpha)$;

- $V$ represents the flow speed.

\section{B. Seawing glider}

As the second validation step, a more complex dynamic manoeuvre is simulated. A Seawing glider is analysed while performing a descending spiralling dive, based on the work detailed in [8]. For this test case, the full set of actuators is used: the VBD is commanded to increase the overall vehicle mass, the shifting actuator is moved towards the front aft of the vehicle and the roll mass is displaced so that the vehicle obtains a constant roll angle. The resulting 3D path can be seen in Figure 5.

The numerical results obtained from the OpenModelica simulator are compared with the ones reported from [8] in Table II. Note that the largest relative error of $15.39 \%$ for the yaw angular rate in fact corresponds to a very small absolute error, thus demonstrating the accuracy of the OpenModelica simulator.

\section{Seaglider parameters identification}

Systematic CFD analyses have been carried out to obtain the hydrodynamic performance of the glider, as illustrated in Figure 6. Environmental and operational conditions are varied, including the angle of attack, sideslip angle and the

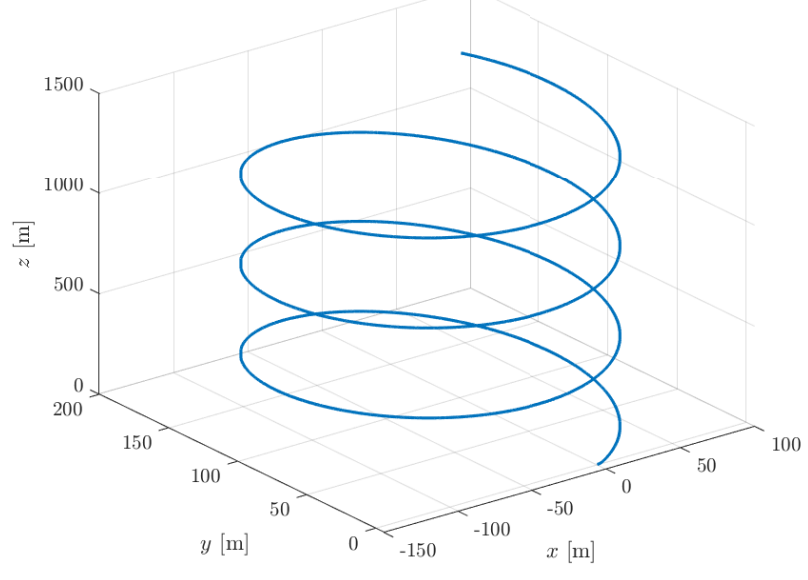

Fig. 5: Seawing spiralling trajectory.

TABLE II: Simulation results comparison.

\begin{tabular}{cccc}
\hline Quantity & $\begin{array}{c}\text { Seawing } \\
\text { reference [8] }\end{array}$ & OpenModelica & Relative error \\
\hline Radius $[\mathrm{m}]$ & 100.83 & 93.88 & $6.89 \%$ \\
Angle attack $\left[{ }^{\circ}\right]$ & 1.267 & 1.258 & $0.71 \%$ \\
Sides angle $\left[{ }^{\circ}\right]$ & -1.283 & -1.396 & $8.81 \%$ \\
$V[\mathrm{~m} / \mathrm{s}]$ & 0.490 & 0.491 & $0.2 \%$ \\
Yaw angular rate $[\mathrm{rad} / \mathrm{s}]$ & 0.0039 & 0.0033 & $15.39 \%$ \\
Pitch angle $\left[{ }^{\circ}\right]$ & -13.703 & -13.011 & $5.05 \%$ \\
Roll angle $\left[{ }^{\circ}\right]$ & -35.641 & -35.643 & $0.01 \%$ \\
\hline
\end{tabular}

operating velocity, so as to derive the desired equations to be incorporated within the simulator.

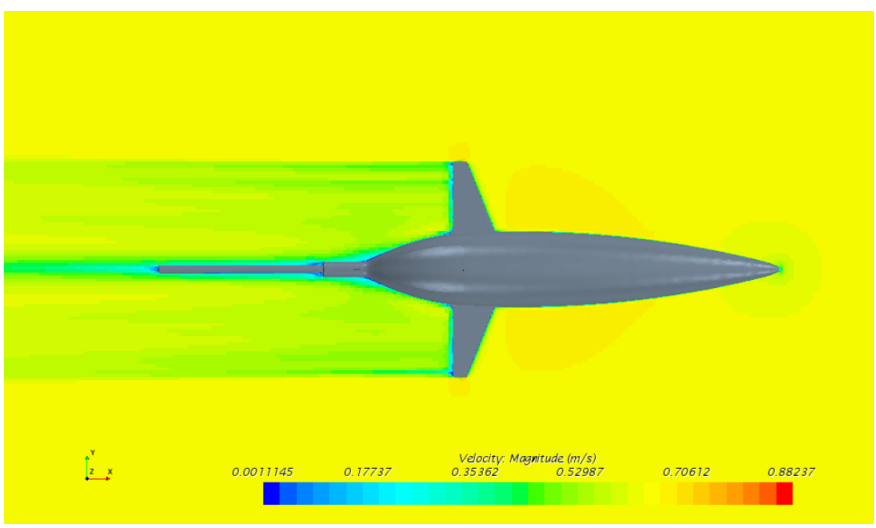

Fig. 6: CFD simulation for Seaglider with $\alpha=\beta=0^{\circ}$, and

$$
V=0.65 \mathrm{~m} / \mathrm{s} \text {. }
$$

The coefficients obtained from the CFD simulation are reported in Tables III, IV and V for the drag, lift and side force, respectively.

These values have been computed for a water density of $\rho=$ $1000 \mathrm{~kg} / \mathrm{m}^{3}$ and a relative velocity between the vehicle to the fluid of $0.5 \mathrm{~m} / \mathrm{s}$. More details related to the CFD model (e.g. governing equations and boundary conditions) can be found in a similar work [15]. The MATLAB function fit has been used 
TABLE III: CFD Results of the Drag force $(V=0.5 \mathrm{~m} / \mathrm{s}$, Unit: N).

\begin{tabular}{|c|c|c|c|c|c|c|}
\hline$\alpha\left[{ }^{\circ}\right]$ & $\mathbf{0}$ & $\mathbf{3}$ & $\mathbf{6}$ & $\mathbf{9}$ & $\mathbf{1 2}$ & $\mathbf{1 5}$ \\
\hline $\mathbf{0}$ & 1.7 & 1.82 & 2.02 & 2.32 & 2.67 & 3.25 \\
\hline $\mathbf{3}$ & 1.85 & 1.97 & 2.16 & 2.45 & 2.81 & 3.38 \\
\hline $\mathbf{6}$ & 2.22 & 2.37 & 2.54 & 2.82 & 3.19 & 3.73 \\
\hline $\mathbf{9}$ & 2.78 & 2.88 & 3.11 & 3.41 & 3.75 & 4.2 \\
\hline $\mathbf{1 2}$ & 3.67 & 3.65 & 3.78 & 3.99 & 4.32 & 4.9 \\
\hline $\mathbf{1 5}$ & 4.56 & 4.57 & 4.6 & 4.8 & 5.19 & 5.6 \\
\hline
\end{tabular}

TABLE IV: CFD Results of the Lift force $(V=0.5 \mathrm{~m} / \mathrm{s}$, Unit: N).

\begin{tabular}{|c|c|c|c|c|c|c|}
\hline$\alpha\left[^{\circ}\right]$ & $\mathbf{0}$ & $\mathbf{3}$ & $\mathbf{6}$ & $\mathbf{9}$ & $\mathbf{1 2}$ & $\mathbf{1 5}$ \\
\hline $\mathbf{0}$ & 0.08 & 0.08 & 0.1 & 0.03 & 0.06 & 0.06 \\
\hline $\mathbf{3}$ & 3.27 & 3.18 & 3.2 & 3.14 & 3.15 & 2.95 \\
\hline $\mathbf{6}$ & 5.91 & 5.9 & 5.91 & 5.84 & 5.52 & 5.4 \\
\hline $\mathbf{9}$ & 6.25 & 6.35 & 6.45 & 6.82 & 6.46 & 6.52 \\
\hline $\mathbf{1 2}$ & 7.72 & 7.1 & 7.38 & 7.68 & 7.63 & 7.72 \\
\hline $\mathbf{1 5}$ & 8.45 & 8.68 & 8.48 & 8.8 & 8.54 & 8.73 \\
\hline
\end{tabular}

to identify the parameters of the hydrodynamic forces reported in Equation (4), and the results are reported in Table VI.

Additionally, three CFD simulations at different speeds are used to validate the quadratic dependency of the drag and lift forces in the quasi-steady state model described by Equation (4). Figure 7 and Figure 8 show the fitting of the drag and lift forces as a function of the vehicle speed, computed with $\alpha=\beta=0^{\circ}$.

Both the drag and the lift forces appear to be well approximated with a quadratic dependency on the speed in the operating glider range $\left(D=7.0343 V^{2.0429}\right.$ and $L=$ $\left.0.3159 \mathrm{~V}^{1.9752}\right)$. The tree different speed points are selected in the standard operating range of the Seaglider, in turn from $0.35 \mathrm{~m} / \mathrm{s}$ to $0.65 \mathrm{~m} / \mathrm{s}$.

The values of the moment of inertia of the vehicle have been computed by assuming the hull as a prolate spheroid with minor semi-axes $a$ and $b$, and major semi-axis $c$. In the case of the Seaglider, $c$ is aligned with the $x_{b}$-axis, $a$ with $y_{b^{-}}$ axis and $b$ with $z_{b}$-axis. Additionally, $a$ and $b$ coincide. Thus, the moment of inertia along the three axes can be expressed

TABLE V: CFD Results of the Side force $(V=0.5 \mathrm{~m} / \mathrm{s}$, Unit: N).

\begin{tabular}{|c|c|c|c|c|c|c|}
\hline$\alpha\left[^{\circ}\right]$ & $\mathbf{0}$ & 3 & 6 & 9 & 12 & 15 \\
\hline $\mathbf{0}$ & 0 & 1.54 & 2.48 & 3.6 & 4.49 & 5.51 \\
\hline 3 & 0.01 & 1.50 & 2.44 & 3.45 & 4.39 & 5.43 \\
\hline 6 & 0 & 1.46 & 2.43 & 3.48 & 4.18 & 5.24 \\
\hline 9 & 0 & 1.35 & 2.26 & 3.19 & 4 & 5.2 \\
\hline 12 & 0.25 & 1.2 & 1.9 & 3 & 3.92 & 5 \\
\hline 15 & 0.16 & 1.16 & 1.85 & 3.06 & 3.87 & 4.62 \\
\hline
\end{tabular}

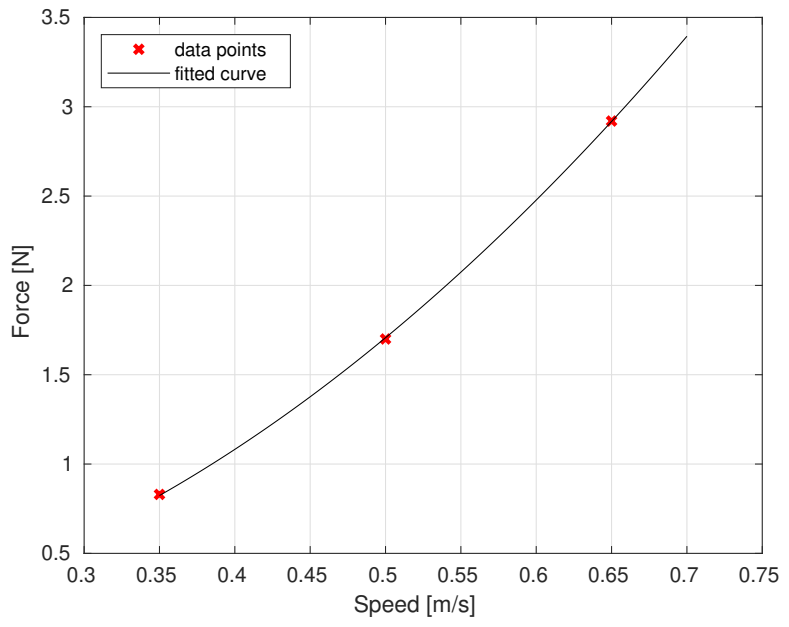

Fig. 7: Drag force vs. surge speed for $\alpha=\beta=0^{\circ}$.

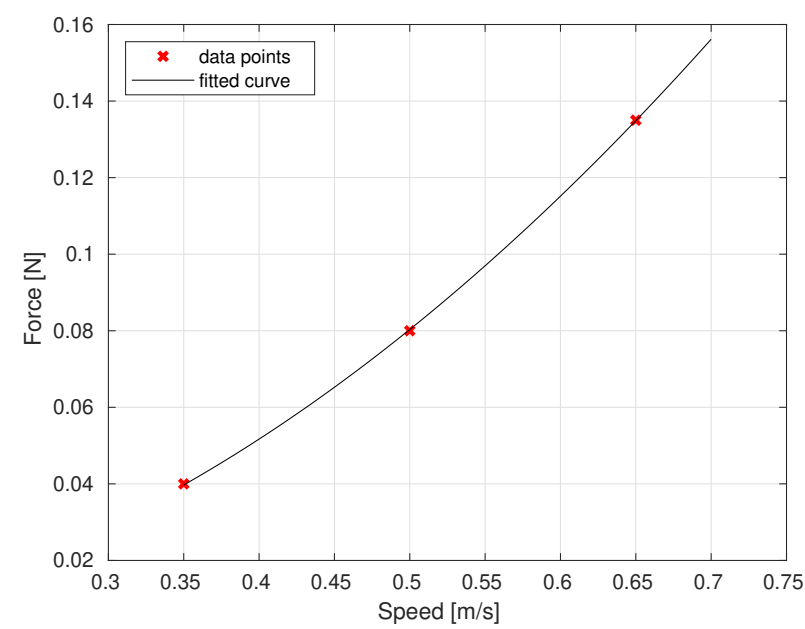

Fig. 8: Lift force vs. surge speed for $\alpha=\beta=0^{\circ}$.

as:

$$
\begin{aligned}
& I_{x x}=\frac{1}{5} m\left(a^{2}+b^{2}\right) \\
& I_{y y}=I_{z z}=\frac{1}{5} m\left(a^{2}+c^{2}\right)
\end{aligned}
$$

where $m$ represents the overall mass of the vehicle.

The values of $a, b$ and $c$ are retrieved from the CAD drawing of the vehicle.

In a similar fashion, the added mass of the vehicle is estimated under the assumption that the hull can be approximated as a prolate spheroid. For such a geometry, the added mass 
coefficients can be computed as reported in [16]:

$$
\begin{aligned}
e^{2} & =1-\frac{b}{a} \\
\alpha_{0} & =\frac{2\left(1-e^{2}\right)}{e^{2}}\left(\frac{1}{2} \log \left(\frac{1+e}{1-e}\right)-e\right) \\
\beta_{0} & =\frac{1}{e^{2}}-\frac{1-e^{2}}{2 e^{2}} \log \left(\frac{1+e}{1-e}\right) \\
k_{1} & =\frac{\alpha_{0}}{2-\alpha_{0}} \\
k_{2} & =\frac{\beta_{0}}{2-\beta_{0}} \\
k^{\prime} & =\frac{e^{4}\left(\beta_{0}-\alpha_{0}\right)}{\left(2-e^{2}\right)\left[2 e^{2}-\left(2-e^{2}\right)\left(\beta_{0}-\alpha_{0}\right)\right]} \\
X_{\dot{u}} & =-k_{1} m \\
Y_{\dot{v}} & =Z_{\dot{w}}=-k_{2} m \\
K_{\dot{p}} & =0 \\
M_{\dot{q}} & =N_{\dot{r}}=-k^{\prime} I_{y y}
\end{aligned}
$$

An open-source utility to compute the added mass coefficients of a prolate spheroid is published in the repository ${ }^{2}$.

The values of the dynamic and geometrical parameters of the Seaglider (ogive model) are summarised in Table VI.

The missing coefficients include the saturation values of the actuators, the initial location of the shifting battery pack as well as the value of its mass.

\section{CONCLUSiOns}

Glider simulators are of particular interest to further understand the dynamics of the vehicles in the highly nonlinear marine environment. Relevant modelling can in turn support the forecast and mitigation of possible faults by developing more robust control strategies, thus the devices are expected to perform better in dealing with instances such as when experiencing unexpected ocean currents or when encountering drifting debris.

In this paper an open-source simulator of AUGs has been proposed and validated by comparing the obtained results with other works available in literature. A final validation step needs to be carried out entailing the comparison of the dynamics computed by the proposed simulation with the one recorded during a real Seaglider deployment. Preliminary studies are reported to identify the dynamic parameters of a Seaglider, based on both CFD analysis and data retrieved from the mission trim and ballast sheet.

\begin{tabular}{|c|c|c|c|}
\hline Parameters & Values & Units & Explanation \\
\hline$L_{t o t}$ & 2.5 & $\mathrm{~m}$ & Total vehicle length \\
\hline$L_{\text {hull }}$ & 1.8 & $\mathrm{~m}$ & Hull length \\
\hline$d$ & 0.3 & $\mathrm{~m}$ & Hull max diameter \\
\hline$m$ & 56.399 & $\mathrm{~kg}$ & Total vehicle mass \\
\hline$\nabla$ & 55.20 & 1 & Nominal volume \\
\hline $\begin{array}{l}{\left[x_{G},\right.} \\
y_{G} \\
\left.z_{G}\right] \\
\end{array}$ & $\begin{array}{c}0 \\
0, \\
0.193]\end{array}$ & $\mathrm{m}$ & $\begin{array}{l}\text { Distance from the } \\
\text { body frame and the } \\
\text { centre of gravity }\end{array}$ \\
\hline $\begin{array}{l}{\left[x_{B}\right.} \\
y_{B} \\
\left.z_{B}\right]\end{array}$ & $\begin{array}{c}0 \\
0 \\
-0.303] \\
\end{array}$ & $\mathrm{m}$ & $\begin{array}{l}\text { Distance from the } \\
\text { body frame and the } \\
\text { centre of buoyancy }\end{array}$ \\
\hline$I_{x x}$ & 0.51 & $\mathrm{~kg} \mathrm{~m}^{2}$ & $\begin{array}{l}\text { Mom. of inertia } \\
\text { around } x_{b} \text {-axis }\end{array}$ \\
\hline$I_{y y}$ & 7.47 & $\mathrm{~kg} \mathrm{~m}^{2}$ & $\begin{array}{l}\text { Mom. of inertia } \\
\text { around } y_{b} \text {-axis }\end{array}$ \\
\hline$I_{z z}$ & 7.47 & $\mathrm{~kg} \mathrm{~m}^{2}$ & $\begin{array}{l}\text { Mom. of inertia } \\
\text { around } z_{b} \text {-axis }\end{array}$ \\
\hline$K_{D 0}$ & 0.0459 & $\mathrm{~kg} / \mathrm{m}$ & Drag force coefficient \\
\hline$K_{D}$ & 9.4377 & $\mathrm{~kg} /\left(\mathrm{m} \mathrm{rad}^{2}\right)$ & Drag force coefficient \\
\hline$K_{\beta}$ & 1.4086 & $\mathrm{~kg} /(\mathrm{m} \mathrm{rad})$ & Side slip force coefficient \\
\hline$K_{L 0}$ & 2.157 & $\mathrm{~kg} / \mathrm{m}$ & Lift force coefficient \\
\hline$K_{L}$ & 4.881 & $\mathrm{~kg} /(\mathrm{m} \mathrm{rad})$ & Lift force coefficient \\
\hline$X_{\dot{u}}$ & -5.94 & $\mathrm{~kg}$ & $\begin{array}{c}\text { Added mass coeff. on } \\
x_{b} \text {-axis generated by } \\
\text { acceleration along } x_{b} \text {-axis }\end{array}$ \\
\hline$Y_{\dot{v}}$ & -46.53 & $\mathrm{~kg}$ & $\begin{array}{c}\text { Added mass coeff. on } \\
y_{b} \text {-axis generated by } \\
\text { acceleration along } y_{b} \text {-axis }\end{array}$ \\
\hline$\overline{Z_{\dot{w}}}$ & -46.53 & $\mathrm{~kg}$ & $\begin{array}{c}\text { Added mass coeff. on } \\
z_{b} \text {-axis generated by } \\
\text { acceleration along } z_{b} \text {-axis }\end{array}$ \\
\hline$K_{\dot{p}}$ & 0 & $\mathrm{~kg} \mathrm{~m}^{2}$ & $\begin{array}{l}\text { Added mass coeff. around } \\
x_{b} \text {-axis generated by } \\
\text { acceleration around } x_{b} \text {-axis }\end{array}$ \\
\hline$M_{\dot{q}}$ & -1.37 & $\mathrm{~kg} \mathrm{~m}^{2}$ & $\begin{array}{l}\text { Added mass coeff. around } \\
y_{b} \text {-axis generated by } \\
\text { acceleration around } y_{b} \text {-axis }\end{array}$ \\
\hline$N_{\dot{r}}$ & -1.37 & $\mathrm{~kg} \mathrm{~m}^{2}$ & $\begin{array}{l}\text { Added mass coeff. around } \\
z_{b} \text {-axis generated by } \\
\text { acceleration around } z_{b} \text {-axis }\end{array}$ \\
\hline
\end{tabular}

Future development of the simulator will comprise embedding environmental disturbances and extending the dynamic model so as to account for depth-related hull expansion phenomena. Additional work will entail interfacing the OpenModelica-based simulator with ROS, so that the real control systems developed in $\mathrm{C}++$ or Python can be tested before the deployment stage in a safely manner.

\footnotetext{
${ }^{2}$ https://github.com/dave-ai/Added-mass-prolate-spheroid
}

TABLE VI: Seaglider dynamic parameters.

\section{REFERENCES}

[1] Phillips, AB and Haroutunian, M and Man, SK and Murphy, AJ and Boyd, SW and Blake, JIR and Griffiths, G, "Nature in Engineering for Monitoring the Oceans: Comparison of the energetic costs of marine animals and AUVs", The Institution of Engineering and Technology, 2012.

[2] Eriksen, Charles C and Osse, T James and Light, Russell D and Wen, Timothy and Lehman, Thomas W and Sabin, Peter L and Ballard, John W and Chiodi, Andrew M, "Seaglider: A long-range autonomous underwater vehicle for oceanographic research", IEEE Journal of oceanic Engineering, vol. 26, no. 4, pp. 424-436, 2001.

[3] Webb, Douglas $\mathrm{C}$ and Simonetti, Paul J and Jones, Clayton P, "SLOCUM: An underwater glider propelled by environmental energy", IEEE Journal of oceanic engineering, vol. 26, no. 4, pp. 447-452, 2001.

[4] Yu, Jian-cheng and Zhang, Ai-qun and Jin, Wen-ming and Chen, Qi and Tian, Yu and Liu, Chong-jie, "Development and experiments of the sea-wing underwater glider", China Ocean Engineering, Springer, vol. 25, no. 4, pp. 721-736, 2001.

[5] Queste, Bastien Y and Heywood, Karen J and Kaiser, Jan and Lee, Gareth A and Matthews, Adrian and Schmidtko, Sunke and WalkerBrown, Christopher and Woodward, Stephen W, "Deployments in extreme conditions: Pushing the boundaries of Seaglider capabilities", IEEE OES Autonomous Underwater Vehicles (AUV), pp. 1-7, 2021.

[6] Graver, Joshua Grady, "Underwater gliders: Dynamics, control and design", PhD Thesis, 2005. 
[7] Fan, Shuangshuang and Woolsey, Craig A, "Dynamics of underwater gliders in currents", Ocean Engineering, Elsevier, vol. 84, pp.249-258, 2014.

[8] Zhang, Shaowei and Yu, Jiancheng and Zhang, Aiqun and Zhang, Fumin, "Spiraling motion of underwater gliders: Modeling, analysis, and experimental results", Ocean Engineering, Elsevier, vol. 60, pp. 1-13, 2013.

[9] Grande, Davide and Bascetta, Luca and Martins, Alfredo, "Modeling and simulation of a spherical vehicle for underwater surveillance", OCEANS 2018 MTS/IEEE Charleston, IEEE, pp. 1-7, 2018.

[10] Fossen, Thor I, "Handbook of marine craft hydrodynamics and motion control", John Wiley \& Sons, 2011.

[11] Fernandez, Ramon A Suarez and Grande, Davide and Martins, Alfredo and Bascetta, Luca and Dominguez, Sergio and Rossi, Claudio, "Modeling and control of underwater mine explorer robot UX-1", IEEE Access, vol. 7, pp. 39432-39447, 2019.

[12] Elmqvist, Hilding, "A structured model language for large continuous systems", PhD Thesis TFRT-1015, Department of Automatic Control, Lund Institute of Technology (LTH), 1978.

[13] Fritzson, Peter and Pop, Adrian and Asghar, Adeel and Bachmann, Bernhard and Braun, Willi and Braun, Robert and Buffoni, Lena and Casella, Francesco and Castro, Rodrigo and Danós, Alejandro and others, "The OpenModelica integrated modeling, simulation and optimization environment", Proceedings of the 1st American Modelica Conference, pp. 8-10, Modelica Association, 2018.

[14] Shuai, SUN and Lei, WANG and Zhiping, LI and Peng, GU and Yuting, FENG and Yujia, SUN, "Modeling and Simulation of Planetary Rover Using Modelica", 2020 Chinese Automation Congress (CAC), pp. $7582-$ 7586, IEEE, 2020.

[15] Rehman, Faheem Ur and Huang, Luofeng and Anderlini, Enrico and Thomas, Giles, "Hydrodynamic Modelling for a Transportation System of Two Unmanned Underwater Vehicles: Semi-Empirical, Numerical and Experimental Analyses", Journal of Marine Science and Engineering, vol. 9, pp. 500, Multidisciplinary Digital Publishing Institute, 2021.

[16] Imlay, Frederick H, "The complete expressions for added mass of a rigid body moving in an ideal fluid", DAVID Taylor Model Basin Washington DC Institution, 1961. 